\title{
El Cirujano de Trauma y Emergencias: Pasado, Presente y
}

\section{Futuro}

\author{
Carlos A Ordóñez
}

\section{El Pasado}

La tasa de homicidios en Cali a principios de la década de los 80, era de 20 por 100.000 habitantes, lo que se considera una epidemia. A mediados de los 80 , la tasa ascendió a 60 por cada 100.0000 habitantes, en una época marcada por movimientos generadores de violencia, como la guerrilla del M19, el inicio del narcotráfico y el de las organizaciones criminales. La combinación de estos fenómenos pudo ser el factor clave que influyera significativamente en el incremento de la criminalidad. Además, se calculaba que, por cada asesinato, se presentaban alrededor de 6 heridos, lo que desembocaría inevitablemente en un aumento de los pacientes víctima de trauma en los servicios de urgencias de los grandes hospitales.

En este mismo momento, en el Hospital Universitario del Valle, Armando González, director general, y Ricardo Ferrada, jefe de Urgencias, tuvieron la idea de formar un grupo de cirujanos que se dedicaran únicamente al manejo del trauma y las urgencias. Antes de esto, los cirujanos dedicados a la cirugía electiva hacían turnos de urgencias como contraprestación dentro de su especialidad. La idea era, precisamente, cambiar esto: que hubiera cirujanos dedicados exclusivamente a manejar esta nueva realidad.

La tasa de mortalidad continuó ascendiendo y, para los años 90, se tuvo el pico más alto, que coincidió con una de las épocas más violentas de Colombia. El auge del conflicto, perpetuado por los carteles del narcotráfico de Medellín y Cali, hizo que la tasa de homicidios aumentara a 124 por cada 100.000 habitantes en 1994. En Cali y Medellín, se alcanzaron cifras superiores a 300 homicidios por cada 100.000 habitantes.

En esta misma época, en 1986, Aurelio Rodríguez y un grupo de cirujanos mexicanos: Manuel Gómez-Palacio, Carlos Moreno, Armando Baqueiro, Alejandro Grife y García-Morales "el Max," tuvieron la idea de crear una sociedad dedicada a la educación del trauma en las Américas. ${ }^{1}$ Fue en Ciudad de México donde se tuvo la idea de crear la Sociedad Panamericana de Trauma. En noviembre de ese mismo año, en el Hotel Tequendama de Bogotá (Colombia), grandes personajes de la cirugía de Colombia y las Américas confirmaron la creación de la sociedad; la idea se forjó en México y se concretó en Bogotá (Fig. 1). La Sociedad Panamericana de Trauma se concibió como una organización que agrupara las tres Américas: Norteamérica, Centroamérica y Suramérica. Su objetivo es forjar lazos y contribuir a la educación, la investigación y el desarrollo de avances en el manejo del trauma y las emergencias, a fin de crear intervenciones destinadas a disminuir la mortalidad y mejorar los resultados en los pacientes que sufren traumatismos.

Durante este periodo de creación y consolidación de la Sociedad, Ricardo Ferrada concretó la idea de que los cirujanos se convirtieran realmente en cirujanos de trauma y emergencias, y comenzó a imprimir esta idea en todos sus discípulos.
Departamento de Cirugía, Fundación Valle del Lili, Universidad del Valle, Cali, Colombia

Corresponding Author: Carlos A Ordóñez, Departamento de Cirugía, Fundación Valle del Lili, Universidad del Valle, Cali, Colombia, Phone: +5723319090, e-mail: ordonezcarlosa@gmail.com

How to cite this article: Ordóñez CA. The Trauma and Emergency Surgeon: Past, Present and Future. Panam J Trauma Crit Care Emerg Surg 2019;8(1):1-11.

Presented at: XXX Congreso Panamericano de Trauma. Ciudad de México, México. Noviembre de 2017

Source of support: Fundacion Valle del Lili

Conflict of interest: None

En Quito (Ecuador) en 1987, fue donde el grupo de residentes de cirugía general de la Universidad del Valle y los cirujanos recién graduados, presentaron por primera vez en un congreso internacional sus trabajos de investigación en relación con el manejo del trauma que se hacía en el Hospital Universitario del Valle de Cali. Paralelamente al crecimiento del trauma en el suroccidente colombiano, en 1984, Oswaldo Borráez colocó la primera "bolsa de Bogotá" en el Hospital San Juan de Dios de Bogotá. ${ }^{2}$ Todos estábamos haciendo lo mismo, pero, en ese entonces, la comunicación era muy difícil y no sabíamos quién realmente lo había hecho primero. En una visita a Bogotá, David Feliciano fue quien le dio el nombre a esta intervención: bolsa de Bogotá (Bogotá bag).

Aurelio Rodríguez y Ricardo Ferrada, en 1988, hicieron un primer convenio para que los cirujanos estadounidenses de Baltimore, Md, y de Israel, vinieran al Hospital Universitario del Valle y, desde Colombia, fueran cirujanos a sus hospitales en Estados Unidos, a compartir experiencias. En 1989, David Raines (jefe de cirugía de trauma en la Virginia Commonwealth University, Richmond, Va) y Dabney R. Yarbrough, fueron invitados por el doctor Ferrada al congreso de urgencias quirúrgicas en Cali, para que hablaran sobre el manejo de los pacientes con trauma en la unidad de cuidados intensivos, de la asistencia respiratoria mecánica y del soporte hemodinámico, como medidas que podrían ayudar a aumentar la supervivencia en estos pacientes gravemente lesionados. En ese entonces, los cirujanos de Colombia que manejaban urgencias no entendían la importancia del cuidado intensivo como soporte en los pacientes con trauma con el fin de mejorar la supervivencia.

Después de esta reunión, tuvimos la oportunidad de visitar la Virginia Commonwealth University en Richmond, Virginia, y el Carolinas Medical Center. Logramos comprobar que era cierto el hecho de que los pacientes traumatizados que estaban en la unidad de cuidados intensivos con asistencia respiratoria mecánica y monitorizados hemodinámicamente tenían menor mortalidad; esto era una realidad. Actualmente, la filosofía del cuidado intensivo

(0) The Author(s). 2019 Open Access This article is distributed under the terms of the Creative Commons Attribution 4.0 International License (https://creativecommons. org/licenses/by-nc/4.0/), which permits unrestricted use, distribution, and non-commercial reproduction in any medium, provided you give appropriate credit to the original author(s) and the source, provide a link to the Creative Commons license, and indicate if changes were made. The Creative Commons Public Domain Dedication waiver (http://creativecommons.org/publicdomain/zero/1.0/) applies to the data made available in this article, unless otherwise stated. 


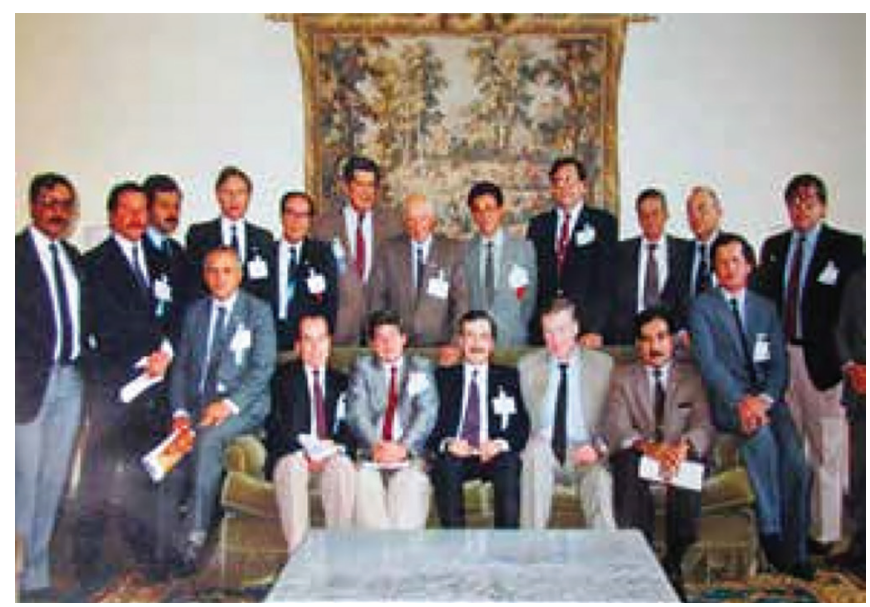

Fig. 1: Profesores del Congreso Avances en Trauma, Shock y Nutrición, Bogotá, 1986

ha sido adaptada a la práctica clínica, pero en aquella época los cirujanos no teníamos la más mínima idea de este concepto. $\mathrm{Ni}$ siquiera conocíamos el concepto de "gasto cardiaco"; de este tamaño era el desconocimiento en cuidados intensivos, y ya éramos cirujanos de emergencias para ese entonces.

Además, tuvimos la fortuna de conocer a Adams Cowley en Baltimore, el fundador del Shock Trauma Center, el creador del concepto "la hora dorada en trauma": "Si puedo llegar a ti, y detener el sangrado, restaurar la presión arterial, en la primera hora después del accidente, entonces, probablemente te puedo salvar". Me permito citarlo, "hemos hecho un gran trabajo", dijo en el recorrido por el nuevo centro, "pero no hemos hecho lo suficiente", "la gente está muriendo innecesariamente". Esta frase la tenemos que tener siempre presente, en cada momento que nos enfrentemos a un paciente con trauma y ver si estamos haciendo bien o debemos cambiar la estrategia para que la gente no muera innecesariamente.

Después de ver todo esto, algunos cirujanos de Cali tuvimos la oportunidad de ir a Bogotá para iniciar nuestra formación en cuidados intensivos y fue en el Hospital San Juan de Dios, bajo la dirección de Alonso Gómez-Duque, donde aprendimos los conceptos de cuidado crítico que luego aplicaríamos en los pacientes traumatizados de Cali.

En los años 90, aprendimos un concepto de suprema importancia: debíamos integrar y estudiar la cirugía del trauma y las emergencias al cuidado intensivo. Entonces, en este año por primera vez, bajo la dirección de Armando González, creamos la primera unidad de cuidado crítico quirúrgico en el Hospital Universitario del Valle. Los pacientes de trauma no podían ir a cuidados intensivos, ese era un privilegio solo para los pacientes médicos con infarto u otras cirugías mayores. En ese entonces, en lo que hoy es un área en la sala de hospitalización de "Cirugía Mujeres" en el Hospital Universitario del Valle, se creó la Unidad de Cuidado Crítico con una donación de Aurelio Rodríguez, quien donó los primeros monitores provenientes del Shock Trauma Center. Además, un paciente atendido por Ricardo Ferrada, que había sido manejado en la nueva Unidad de Cuidados Intensivos, donó otros respiradores y, con esto, empezamos nuestra primera unidad quirúrgica de cuidados intensivos desde cero.

¿Qué sucedió? En ese entonces, Armando González, jefe del Departamento de Cirugía, clasificaba los pacientes con una tarjeta para identificarlos, verde: paciente no crítico, roja: paciente crítico; el paciente crítico tenía una mortalidad entre el 65 y el 75\%. Un año después de la implementación de la unidad de cuidado crítico, la mortalidad bajó al 35\%. En ese entonces, no había terapia respiratoria en las noches ni una buena monitorización cardiovascular, pero a pesar de tener falencias, se logró una disminución clínicamente significativa. Por lo anterior, el Departamento de Cirugía tomó la decisión de que los cirujanos fueran a estudiar formalmente Cuidados Intensivos a Bogotá. Esto fue un cambio radical, un aporte gigante a la educación de los cirujanos hecho por el Departamento de Cirugía de la Universidad del Valle y del Hospital Universitario en Cali.

Estábamos atrasados más de 20 años en el manejo de cuidados intensivos con respecto al mundo. Pusimos nuestro primer catéter de Swan-Ganz en Cali en 1990, 20 años después de que se hubiera puesto el primero en Estados Unidos ${ }^{3}$ y 10 años después del primero en Bogotá.

Hoy, la Fundación Valle del Lili se consolida como el hospital número uno en Colombia, con una unidad de cuidados intensivos que cuenta con más de 100 camas, con una mortalidad menor del $10 \%$ de los pacientes con trauma. Esto es gracias a todo el desarrollo en tecnología y educación. Nuestra unidad de cuidado crítico es comparable con otras de alta complejidad en otras partes, como Norteamérica o Europa. Esto demuestra que las grandes cosas sí se pueden hacer, si se toma la decisión correcta en el momento adecuado y se tiene el liderazgo para hacerlo.

Volvamos atrás de nuevo. David Feliciano visitó Cali en 1991, estuvo tres semanas en el Hospital Universitario del Valle operando con los residentes y cirujanos, y compartió su experiencia y conocimiento con nosotros, lo cual nos fortaleció como equipo. Esto sirvió para consolidar el grupo de cirujanos de trauma del Hospital, porque personas como él comenzaron a integrar a los cirujanos y compartir el conocimiento, y es así como, en ese mismo año, se realizó el primer congreso de la Sociedad Colombiana de Trauma con el aval de la Sociedad Panamericana de Trauma.

Entendimos que la gravedad del daño anatómico y la gravedad del daño fisiológico se pueden integrar en un concepto denominado 'cirugía de control de daños'. En 1993, en el congreso de la Asociación Colombiana de Cirugía, en Bogotá, Feliciano habló sobre técnicas de control de daños, lo que era una idea relativamente nueva para nosotros $y$, aunque ya lo veníamos realizando en cierta medida, fue Feliciano quien nos dijo cómo se debería llamar y cómo se debe hacer. Fue en ese momento cuando se empezó a acuñar el nombre de "cirugía de control de daños", descrito por primera vez en 1993 por Rotondo y Schwab. ${ }^{4}$

Fue así como, de 1992 a 2016, se realizaron intercambios y rotaciones con cerca de 500 residentes y cirujanos visitantes de países como Argentina, Brasil, Cuba, Ecuador, Perú, México, Estados Unidos, Israel, Japón, Francia e Inglaterra, en convenio con la Universidad del Valle, en el Hospital Universitario del Valle. Esto ha permitido fortalecer las relaciones con los profesores asistentes al congreso de la Sociedad Panamericana de Trauma, que se ha venido realizando durante estos últimos 30 años.

Veinte años después de la aparición de la bolsa de Bogotá, en el año 2004, Rao Ivatury visitó la ciudad de Cali y nos enseñó a colocar el primer sistema de presión negativa, vacuum pack. ${ }^{5}$ Llevábamos cuatro años intentando usarlo en la práctica diaria en la Fundación Valle del Lili, pero no habíamos dado el paso definitivo y lo logramos gracias a la visita de Ivatury a nuestro centro. Este es otro ejemplo de la importancia del intercambio, el estímulo y las enseñanzas que se difunden a través de la Sociedad Panamericana de Trauma y el 
intercambio transnacional con cirujanos de todo el mundo. Desde ese año, cambiamos el manejo del abdomen abierto radicalmente.

Con esto podría resumir nuestro pasado que, creo, es el pasado compartido por muchos grupos de cirugía de trauma en Latinoamérica, con hitos tan fundamentales como el inicio del cuidado intensivo quirúrgico, la cirugía de control de daños y el manejo del abdomen abierto.

\section{El Presente}

¿Qué está pasando con la cirugía general? ¿Está desapareciendo? En Suramérica, los cirujanos generales perdimos el papel del manejo de las enfermedades quirúrgicas. El campo de acción del cirujano general ha venido siendo mermado, ya que cada subespecialidad ha tomado el manejo de cada una de sus enfermedades. Tener cirujanos con conocimiento y experiencia en un área específica es un gran avance y un beneficio para los pacientes. Sin embargo, esto implica que, al mismo tiempo, se ha venido reduciendo el papel del cirujano general, y en algunos países está muriendo más rápido que en otros. Más aún, en las grandes ciudades esto es una realidad.

En Estados Unidos, la cirugía general también ha venido desapareciendo y el papel de la cirugía de trauma se estaba limitando, ya que muchas de las nuevas herramientas del manejo de los heridos consisten en técnicas selectivas no operatorias. En 2005, se definió el concepto de "acute care surgery" como una nueva especialidad, y así cambió el panorama en Norteamérica. ${ }^{6}$ Mientras esto sucedía, otras grandes cosas pasaron. La Sociedad Panamericana de Trauma seguía integrando a sus miembros; por ejemplo, en el 2006, Juan Carlos Puyana de la Universidad de Pittsburgh, en conjunto con la Universidad del Valle, logró obtener una subvención en investigación de Fogarty por USD\$750.000 destinados a la educación en trauma. Con esto, Álvaro Sánchez hizo un doctorado en ciencias traslacionales. ${ }^{7}$ Además, Alberto García y Carlos Ordóñez, entre otros, tuvimos la fortuna de visitar Pittsburgh, y conocer cómo se maneja y se produce la investigación en trauma. En total, nueve personas del área tuvieron la oportunidad de ir y capacitarse en investigación.

Desde ese año, nos pusimos una meta que se ha venido cumpliendo a cabalidad: hacer presentaciones orales y en carteles de manera consecutiva en el congreso de la American Association for the Surgery of Trauma (AAST), el congreso más importante en trauma

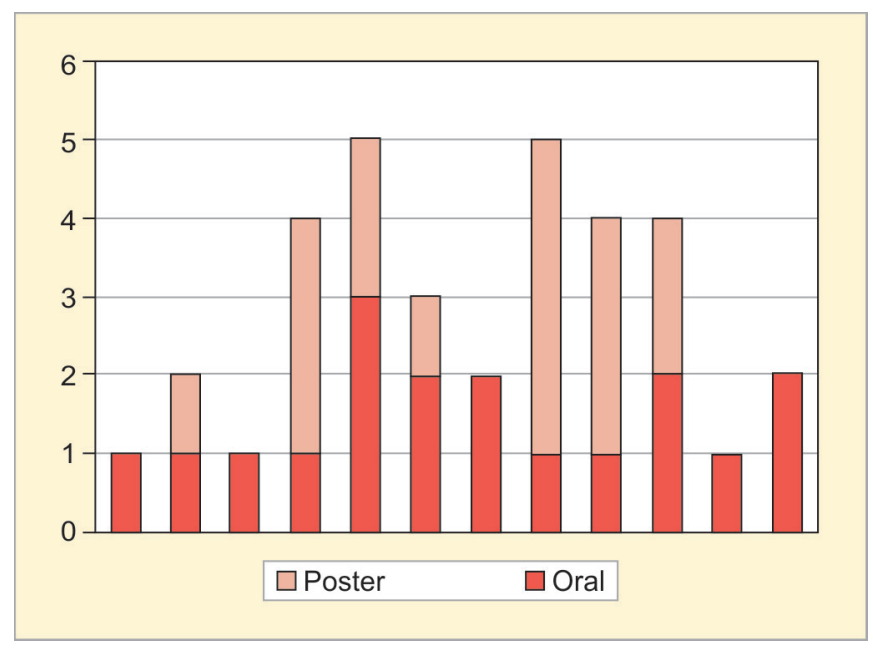

Fig. 2: Tendencia en el número de trabajos científicos presentados en el Annual Meeting of the American Association for the Surgery of Trauma (AAST) (poster y oral) de los Estados Unidos y del mundo, mostrando nuestra experiencia y aportes al manejo de la cirugía del trauma en Colombia (Fig. 2).

En el 2005, el Comité de Trauma del American College of Surgeons (AAST y ACS) formalizó la iniciativa de crear cirujanos de "acute care surgery" $y$, más adelante en el 2008, entre estas dos instituciones se inició de manera formal el fellowship en Acute Care Surgery. ${ }^{6}$

Paralelamente, en Cali, en el 2007 y con la Universidad del Valle, realizamos la presentación formal del currículo del programa de "Cirugía de Trauma y Emergencias" que se inició en el 2010 con el primer fellow en cirugía de trauma y emergencias de una manera formal. El de Cirugía de Trauma y Emergencias es un programa de segunda especialización, que requiere tener el título de cirujano general como primera especialización. En conjunto con la Universidad del Valle, los dos hospitales que han dado todo el soporte y respaldo para llevar a cabo el programa son: el Hospital Universitario Fundación Valle del Lili y el Hospital Universitario del Valle "Evaristo García".

Los pilares del programa de Cirugía de Trauma y Emergencias son los mismos que los del programa de "Acute Care Surgery" en Estados Unidos. El programa se fundamenta en el trauma, en toda su extensión, la cirugía de urgencias, el manejo del paciente en cuidados intensivos, la cirugía general electiva y, ahora, el nuevo concepto de "cirugía de rescate", que abarca a los pacientes agudos, complicados y no traumatizados de otras especialidades, que los cirujanos de trauma y emergencias están en capacidad de manejary salvar (Fig. 3). ${ }^{8}$

Los primeros cirujanos en recibir el título de cirujanos de trauma y emergencias fueron Carlos A. Ordóñez, Laureano Quintero y Ricardo Ferrada, como profesores del Departamento de Cirugía de la Universidad del Valle, y dos fellows formales, Luis Fernando Pino y Juan Carlos Salamea.

¿Qué tiene de especial y diferente un cirujano de trauma y emergencias con respecto a un cirujano general? Tiene todas las habilidades en el manejo quirúrgico del trauma, de la unidad de cuidados intensivos, de la cirugía de cuidado agudo, de las habilidades docentes para enseñar en su entorno y su país, y de transmitir el conocimiento; además, tiene las habilidades en investigación para generar nuevo conocimiento para el mundo, y el liderazgo para construir un servicio de trauma y emergencias.

Un pilar fundamental del cirujano de trauma y emergencias es el desarrollo de habilidades en investigación. La Sociedad Panamericana de Trauma tiene un excelente registro de trauma, que se ha renovado y está disponible para todos los interesados en investigar con los datos ahí consignados. Cabe destacar el importante papel que tienen en la producción de este registro, Rao Rao Ivatury y Michel B. Aboutanos, de dicha Sociedad. Dicho registro

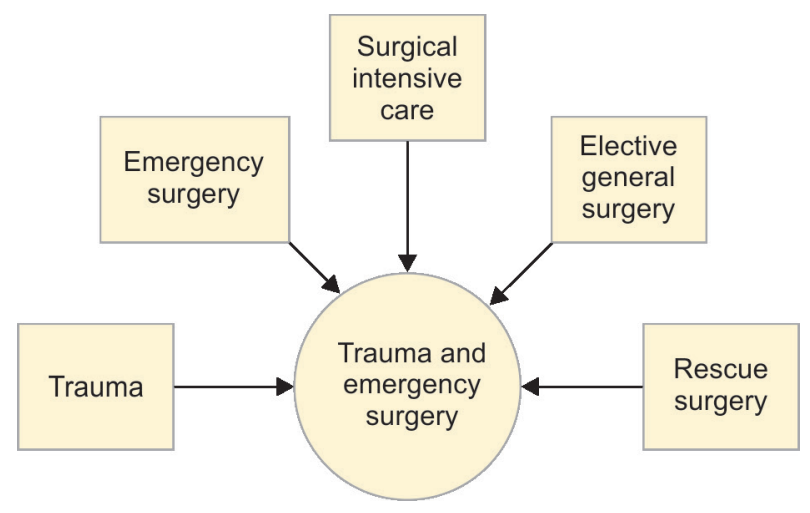

Fig. 3: Pilares de la cirugía de trauma y emergencias 
es una herramienta muy valiosa para producir investigación: la sociedad la provee a todos los interesados.

Para lograr una profunda y adecuada apropiación de la investigación, se necesita de esfuerzos colaborativos. Por ejemplo, desdeel año 2011, Michael Parra ha estado colaborando continuamente desde Florida con nuestro equipo en la conceptualización y la escritura de los trabajos. Este tipo de alianzas son importantes, ya que nos han permitido entender que, para poder transmitir nuestras ideas, necesitamos primero de alguien que las entienda dentro de nuestro contexto latino, pero que, simultáneamente, tenga la capacidad de transmitirlas en otro idioma sin cambiar la esencia de lo que se quiere comunicar. También, desde el 2013, la World Society of Emergency Surgery, realiza de manera continua estudios multicéntricos y guías de manejo en los que convocan a cirujanos de todas partes del mundo para que cada uno, desde su experiencia, aporte a la realización de las guías. Gracias a estas alianzas, hemos tenido la oportunidad de participar y publicar en esta importante sociedad mundial.

Todo esto nos ha dado frutos en investigación y publicaciones. Desde que empezamos a conformar las alianzas estratégicas, el número de publicaciones ha aumentado progresivamente y la calidad de los manuscritos es mejor, lo que nos ha permitido publicar en revistas que se difunden a nivel mundial. Vemos esto como una oportunidad de transmitir el conocimiento a cirujanos ubicados en cualquier parte del mundo, gracias a que tenemos un volumen de trauma que permite hacer estudios que no son viables en otros países. Si somos capaces de convertir la experiencia de los cirujanos en ciencia, y que esta sirva para la educación de la cirugía en el trauma y las emergencias, habremos cumplido una gran parte de la tarea por la que trabajamos, porque ese es uno de los fines que tenemos que cumplir (Fig. 4).

El Hospital Universitario del Valle y la Fundación Valle del Lili son los dos hospitales universitarios que han brindado el soporte al programa de Cirugía de Trauma y Emergencias; además, están brindado apoyo económico para los fellows, ya que pueden recibir honorarios al hacer turnos en cuidados intensivos y cirugía general, como una manera de promover la estabilidad económica mientras terminan su proceso de entrenamiento.

En Pittsburgh, con el apoyo de Juan Carlos Puyana y Andrew B. Peiztman, se iniciaron las rotaciones en Estados Unidos para los fellows de Cirugía de Trauma y Emergencias. Juan Carlos Salamea y Luis Fernando Pino (fellows en ese entonces) fueron los primeros que tuvieron la oportunidad de ir a estos sitios de rotación y, en Richmond, a la Virginia Commonwealth University con Rao Rao Ivatury y Michel B.
Aboutanos. Este soporte ha sido posible en Estados Unidos gracias a la Sociedad Panamericana de Trauma. Años atrás, el tener la oportunidad de hacer rotaciones internacionales durante nuestra formación como cirujanos era algo impensable, por los costos que eso representaba.

En Richmond, Va, el director del sistema de ambulancias, Roberth Lawrence, le ofrece un espacio a los fellows para que tengan la oportunidad de ir y conocer cómo es el manejo del sistema de emergencias médicas. Esto también, gracias a los convenios hechos por medio de la Sociedad Panamericana de Trauma.

Con Michael Parra, tienen la oportunidad de recibir formación en cirugía laparoscópica en trauma y cirugía robótica. El grupo de Gregory Peck (Rutgers University) en New Jersey, nos apoya con programas de formación en trauma. Thomas Scalea, en el Shock Trauma Center de Baltimore, Md, le ha abierto las puertas a nuestros fellows. Generalmente, un programa de rotación semanal cuesta, aproximadamente, USD\$5.000, pero gracias a nuestra integración con esta Sociedad, la rotación es totalmente gratuita.

Tenemos ya en total 28 fellows entre los ya graduados y los activos; de Colombia, 20; de Ecuador, 5; de México, 1; de Chile, 1, y de Costa Rica, 1. Esperamos poder contar con todos los países. Necesitamos que se creen nuevas escuelas similares en todos los países que tengan los recursos. Nuestro grupo está totalmente dispuesto a colaborar y a fomentar este proceso; pronto tendremos una nueva escuela en Medellín, y estamos en el trabajo de promover la creación de escuelas de trauma en Brasil.

Nuevamente, cito a Juan Carlos Salamea, que se formó en nuestra escuela y ahora tiene un grupo consolidado en Ecuador, un Servicio de Cirugía de Trauma y Emergencias y, además, tiene una de las ligas de trauma más importantes de Latinoamérica.

\section{El Futuro}

En las Américas, según el World Economic Forum, la tendencia de la tasa de homicidios por cada 100.000 habitantes, irá en aumento en la década de 2020 a 2030, y para el 2030, incluso, a 40 por cada 100.000 habitantes, una tasa que está muy por encima de las de continentes como África o Asia. Tenemos que prepararnos para enfrentar este reto (Fig. 5).

Nuestra estrategia desde lo pragmático es salvar vidas, parando el sangrado a tiempo: "stop the bleeding on time".

Tenemos que innovar, adelantarnos al futuro con las nuevas intervenciones que existen para el control del sangrado: salas

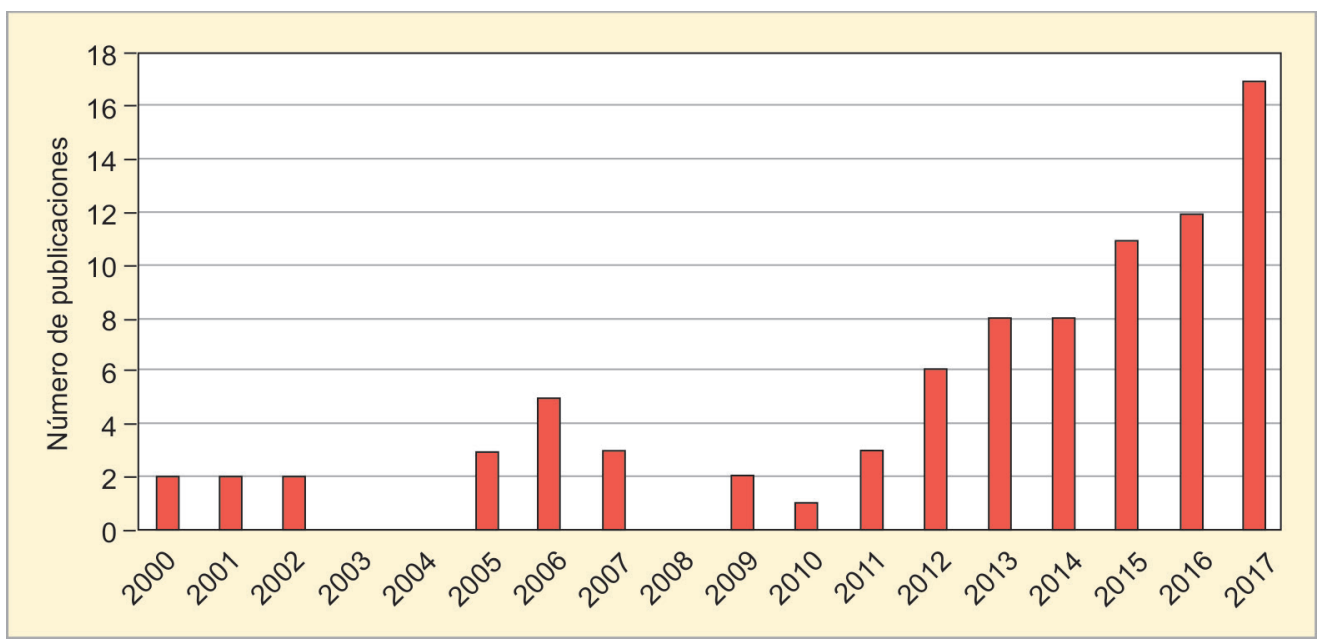

Fig. 4: Tendencia del número de publicaciones en revistas indexadas por año 


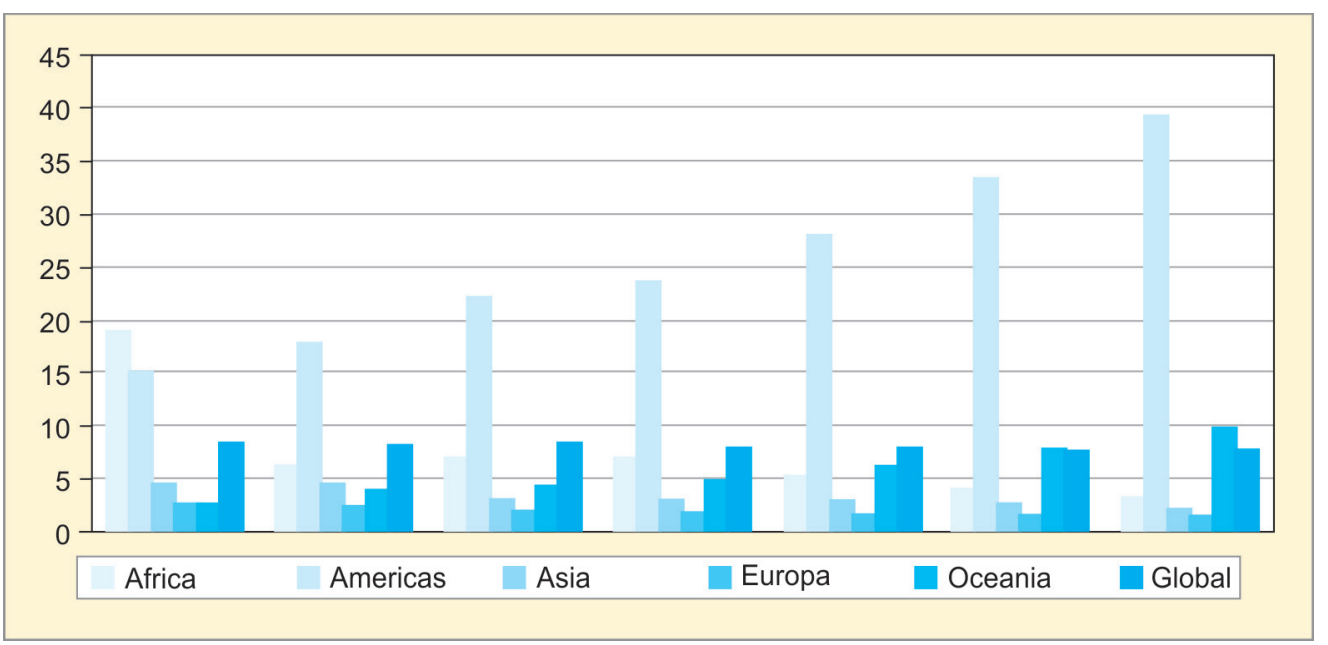

Fig. 5: Proyección de la tasa de homicidios en las Américas, 2000 to 2030: tasa de homicidio por 100.000

híbridas, manejo intravascular, cirugía de rescate y control de daños que no estén relacionados con el trauma, combinados con habilidades en investigación, para poder transmitir rápidamente el conocimiento y lograr cada vez más avances (Fig. 6).

Un claro ejemplo de la aplicación de la innovadora estrategia "stop the bleeding on time", fue el de un paciente de la Fundación Valle del Lili, con trauma hepático complejo, el cual se benefició de intervenciones híbridas. Atendido por un equipo entrenado en trauma, y en estado de inestabilidad, se le practicó una tomografía computarizada y, con un diagnóstico claro, de inmediato se sometió a angiografía y se emboliza la arteria hepática derecha; como seguía en choque, se le colocó un Resuscitative Endovascular Balloon Occlusion of the Aorta (REBOA), a pesar de lo cual continuó con inestabilidad hemodinámica; entonces, se colocó otro Resuscitative Endovascular Balloon Occlusion en la vena cava y se le practicó una laparotomía. ${ }^{9}$ Con toda la seguridad de que teníamos controlado el sangrado de la arteria hepática, pinzadas la aorta y la cava, y con aislamiento hepático, tendríamos más probabilidad de éxito en la cirugía. Hacia esta estrategia de innovación es que queremos apuntar.

He tenido la oportunidad de compartir con cirujanos de trauma en Japón. Allá se implementó una sala híbrida en el servicio de urgencias, y en los estudios se ha demostrado una disminución significativa de la mortalidad de los pacientes atendidos en estas

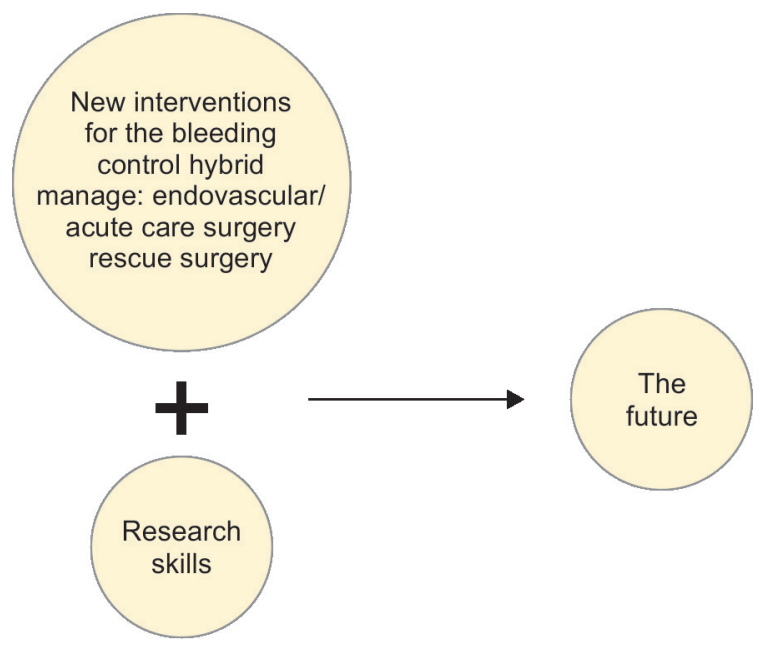

Fig. 6: Habilidades del cirujano de trauma y urgencias del futuro salas frente a las convencionales. La cirugía general tiende a desaparecer, pero la cirugía de trauma nace con nuevas estrategias como estas. Estos son los cirujanos de trauma y emergencias que estamos formando en el momento: con habilidades técnicas en cirugía de trauma, emergencias médico-quirúrgicas, cirugía de rescate, cirugía electiva; con conocimiento profundo del cuidado intensivo quirúrgico; y con la capacidad de educar a generaciones futuras, investigar y producir conocimiento científico, liderar y construir centros de trauma y emergencias.

Ahora tenemos una nueva idea para mejorar aún más la formación de los futuros cirujanos de trauma y emergencias. Juan Pablo Herrera estudió tres años en investigación con el grupo de Adil Haider en Boston, Ma, mediante una alianza estratégica con la Sociedad Panamericana de Trauma. Actualmente, ha tenido la oportunidad de presentar trabajos de trauma en la AAST, sin ser todavía cirujano. La idea es que, al finalizar este primer proceso, regrese a hacer su residencia de cirugía general y, posteriormente, sea fellow de cirugía de trauma y emergencias. Hoy estamos preparando a los cirujanos de trauma como se ha descrito, pero, en el futuro, queremos prepararlos dándoles esta herramienta adicional que será un gran logro en la educación de los futuros cirujanos de trauma y emergencias. Ramiro Manzano, igualmente, ha tenido la oportunidad de presentar trabajos en congresos de la AAST y el American College of Surgeons; él será quien continuará el proceso que ahora está finalizando el doctor Herrera.

El cirujano de trauma y emergencias debe dominar todas las técnicas quirúrgicas, tener la capacidad de investigar y convertir lo aprendido en educación e innovación. Ellos serán los nuevos MacGyver del mundo, capaces de enfrentarse a todo, con el claro objetivo de salvar vidas en sus hospitales y en el mundo. Esta es la meta en la que ahora estamos trabajando, que sean ellos quienes en un futuro estén en capacidad de reemplazar a todos los cirujanos que estamos viviendo hoy el presente (Fig. 7).

Esta conferencia está dedicada a Ricardo Ferrada, quien inició el proceso de la creación de cirujanos de trauma y emergencias en Latinoamérica; a Rao Rao Ivatury, por haber entregado parte de su vida a fomentar esta verdadera unión de las Américas mediante la Sociedad Panamericana de Trauma; a Aurelio Rodríguez, creador de la Sociedad Panamericana de Trauma para lograr la integración que tenemos hoy, y a toda nuestra gran organización, que son los miembros y el alma de lo que hoy es la Sociedad Panamericana de Trauma. 


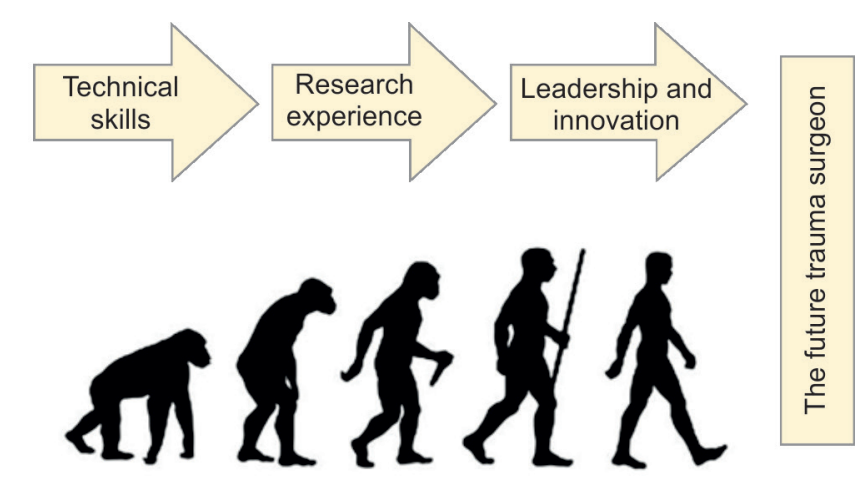

Fig. 7: Modelo de la evolución para llegar a ser el cirujano de trauma y urgencias del futuro

\section{Referencias}

1. Ivatury RR, Aboutanos M. Panamerican trauma society: the first three decades. J Trauma Acute Care Surg 2017;82:966-973. DOI: 10.1097/ TA.0000000000001393.

2. Borráez O. Abdomen abierto: la herida más desafiante. Rev Colomb Cir 2008;23:204-209.
3. Chatterjee K. The Swan-Ganz catheters: past, present, and future. Circulation 2009;119:147-152. DOI: 10.1161/CIRCULATIONAHA. 108.811141.

4. Rotondo MF, Schwab CW, et al. Damage control: an approach for improved survival in exsanguinating penetrating abdominal injury. J Trauma 1993;35:375-383. DOI: 10.1097/TA.0b013e3181e12b3a.

5. Barker DE, Kaufman HJ, et al. Vacuum pack technique of temporary abdominal closure: a 7 year experience with 112 patients. J Trauma Acute Care Surg 2000;48:201-207.

6. Davis KA, Jurkovich GJ. Fellowship training in acute care surgery: from inception to current state. Trauma Surg Acute Care Open 2016;1(1):e000004. DOI: 10.1136/tsaco-2016-000004.

7. Sánchez-Ortiz A. Mortality and costs from acute care surgical emergencies. University of Pittsburgh, 2010. Fecha de consulta: Septiembre de 2017. Disponible en: http://d-scholarship.pitt. edu/25071/1/SanchezAI_ETD_Pitt2015.pdf.

8. Kutcher ME, Sperry JL, et al. Surgical rescue: the next pillar of acute care surgery. J Trauma Acute Care Surg 2017;82:280-286. DOI: 10.1097/ TA.0000000000001312.

9. Ordóñez CA, Herrera-Escobar JP, et al. A severe traumatic juxtahepatic blunt venous injury. J Trauma Acute Care Surg 2016;80:674-676. DOI: 10.1097/TA.0000000000000979. 


\title{
The Surgeon of Trauma and Acute Care Surgery in Latin America: Past, Present, and Future
}

\author{
Carlos A. Ordoñez
}

\section{The Past}

The homicide rate in Cali during the 1980s was 20 per 100,000 inhabitants and was considered an ensuing epidemic. By the mid1980 s, the rate increased to 60 per 100,0000 inhabitants, a time marked by senseless violence from leftist guerrilla groups like the M-19, the drug cartels, and ultra-right para-militia. This perfect storm basically held captive a nation for more than a decade. Also, it has been estimated that for each life lost, six other innocent bystanders were injured. Inevitably, this leads to an increase in patient volume that fell upon the shoulders of an already overburdened network of hospitals throughout the country. The death rate continued to rise, and, by the mid-1990s, it reached its peak, creating one of the most violent times in the Colombian history ( 124 homicides per 100,000 inhabitants in 1994). Cities like Cali and Medellín experienced rates even higher (300 homicides per 100,000 inhabitants).

During this time, Dr Armando González (hospital director) and Ricardo Ferrada (chief of the emergency room (ER)) at Cali's University Hospital introduced the idea of forming a group of surgeons dedicated solely to the management of trauma and emergencies. Before this, penetrating injuries, explosions, and elective surgery were all being managed by general surgeons. The idea was, precisely, to change this and have dedicated surgeons exclusively to handle this new reality.

In 1986, Aurelio Rodríguez and a group of Mexican surgeons, Manuel Gómez-Palacio, Carlos Moreno, Armando Baqueiro, Alejandro Grife, and García-Morales, had the idea of creating a society dedicated to trauma education in the Americas which they called the Panamerican Society of Trauma (PTS). ${ }^{1}$ In November of that same year, in the Tequendama Hotel located in Bogotá, Colombia, many surgeons from Latin and North America came to a consensus and officially created the Society (Fig. 1). The newly created Panamerican Society of Trauma was conceived as an organization that would bring together all three Americas: North, Central,

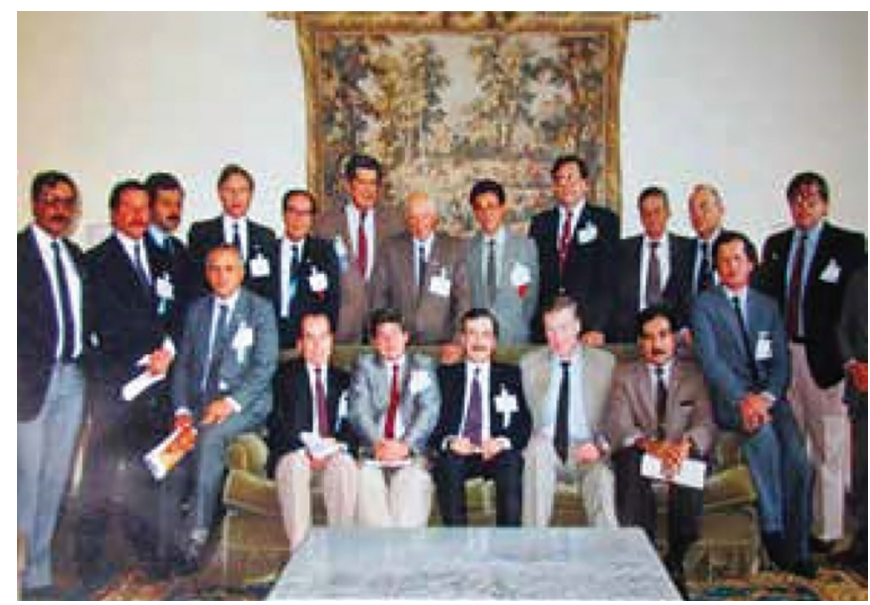

Fig. 1: Professors at the Trauma, Shock and Nutrition Advances Congress. Bogotá 1986.
Department of Surgery, Fundación Valle del Lili, Universidad del Valle, Cali, Colombia

Corresponding Author: Carlos A Ordóñez, Department of Surgery, Fundación Valle del Lili, Universidad del Valle, Cali, Colombia, Phone: +5723319090, e-mail: ordonezcarlosa@gmail.com

How to cite this article: Ordóñez CA. The Surgeon of Trauma and Acute Care Surgery in Latin America: Past, Present, and Future. Panam J Trauma Crit Care Emerg Surg 2019;8(1):1-11.

Presented at: XXX Pan-American Trauma Congress, Mexico city, Mexico. November 2017

Source of support: Fundacion Valle del Lili

Conflict of interest: None

and South America. The goal was to forge ties and promote education and research in the area of trauma.

In 1984, Dr Oswaldo Borráez placed the first "Bogota bag" at the San Juan de Dios Hospital in Bogotá. ${ }^{2}$ On a visit to Bogotá, Dr David Feliciano was the one who gave the name to this brilliant idea. In 1987, in Quito (Ecuador), a group of residents from the University del Valle presented for the first time in an international congress their research work in the management of trauma. Dr's Aurelio Rodríguez and Ricardo Ferrada, in 1988, created an agreement between North American surgeons from Baltimore and also surgeons from Israel to visit the University Hospital Del Valle in Cali, Colombia. In return, Colombian surgeons visited hospitals in the United States with the aim of sharing their experiences. In 1989, Dr David Raines (the head of trauma surgery at Virginia Commonwealth University, Richmond, VA) and Dr Dabney R. Yarbrough were invited by Dr Ferrada to the congress on surgical emergencies in Cali to talk about the management of patients with trauma in the intensive care unit, especially on mechanical ventilation and hemodynamic support. Specific management measures that could help increase survival in these severely injured patients were discussed. At that time, Colombian surgeons who handled emergencies did not appreciate the importance of critical care of trauma patients in the ICU could improve survival.

After this meeting, we had the opportunity to visit the Virginia Commonwealth University in Richmond, Virginia, and the Carolinas Medical Center. We managed to confirm that it was true that traumatized patients that were in the ICU with mechanical ventilation and hemodynamic monitoring had lower mortality. At present, the philosophy of intensive care had been adapted to clinical practice, but at that time, the surgeons in Latin America were unaware of this concept. We were introduced to new concepts such as "cardiac output" and the "the golden hour in trauma." Some surgeons from Cali had the opportunity to go to Bogotá and earn intensive care training at the San Juan de Dios Hospital, under the direction of Dr Alonso Gómez-Duque, where we learned the concepts of critical care that we would then apply to patients in Cali.

In the 1990s and under the direction of Armando González, we created the first surgical critical care unit in the University Hospital in Cali. Prior to this, trauma patients generally were never transferred to the ICU, a privilege only reserved for patients suffering from an $\mathrm{MI}$ or 
surgical patients undergoing major elective procedures. The critical care unit was created with a donation from Dr Aurelio Rodríguez, who donated the first monitors from the Shock Trauma Center, Baltimore. In addition, a patient of Dr Ricardo Ferrada donated respirators and, with this, we started our first intensive care surgical unit.

At that time, Dr Armando González, the head of the Department of Surgery, classified the patients with a card to identify them: green $=$ noncritical and red $=$ patient critical. The critical trauma patient had a mortality between 65 and $75 \%$ and 1 year after the implementation of the critical care unit, the mortality dropped to $35 \%$. The Department of Surgery consequently made the decision that all their surgeons were going to formally study intensive care. This was a radical change, a giant contribution to the education of the surgeons made by the Department of Surgery of the Universidad del Valle and the University Hospital in Cali. We were behind by more than 20 years in intensive care management with respect to the world. We put our first Swan-Ganz catheter in Cali in 1990, 20 years after what would have been the first in the United States. ${ }^{3}$ Today, the Valle del Lili Foundation is considered as one of Colombia's leading hospitals, with an intensive care unit that has more than 100 beds and an overall mortality less than $10 \%$ among trauma patients. This is thanks to all the developments in technology and education. Our critical care unit is comparable with others of high complexity in other parts of the World. This shows that great things can be done if the right decision is made at the right time and with the right leadership.

Let us go back again. Dr David Feliciano visited Cali in 1991 and spent 3 weeks at the University Hospital del Valle operating with residents and surgeons. He shared his experience and knowledge with us, which strengthened us as a team. This served to consolidate the group of trauma surgeons in Cali, because people like him started to integrate surgeons and share knowledge, and, that is how, in that same year, the Colombian Association of Trauma was created and endorsed by the Panamerican Society of Trauma.

We understood that the severity of anatomical damage and the severity of the physiological injury to a patient could be integrated into a management concept called "Damage Control." In 1993, at the Congress of the Colombian Association of Surgery in Bogotá, Dr Feliciano spoke about techniques of damage control. This was a relatively new idea to us and, although already we were doing it to a certain extent, it was Feliciano who told us how it should be done. It was at this same time when the name of "Damage Control Surgery" was described for the first time in 1993 by Rotondo and Schwab. ${ }^{4}$ That is how, from 1992 to 2016 , exchanges and rotations with about 500 residents and visiting surgeons from countries like Argentina, Brazil, Cuba, Ecuador, Peru, Mexico, United States, Israel, Japan, France, and England, in agreement with Universidad del Valle, took place in the University Hospital del Valle. This has allowed us to strengthen relations with scholars around the World for the past 30 years.

In 2004, Dr Rao Ivatury visited the city of Cali and taught us how to place the first negative pressure system "vacuum pack." ${ }^{5}$ This is another example of the importance of intellectual exchange, encouragement, and teachings that are disseminated through the Panamerican Society of Trauma. With this example, we could summarize the past shared by many groups of trauma surgery in Latin America.

\section{The Present}

What is happening with general surgery? Is it disappearing? In South America, the field of action of the general surgeon has been depleted, since each subspecialty has taken the management of each of their diseases away from them. This implies that the role of the general surgeon in many countries is dying faster than in others. In the United States, general surgery also has been disappearing, and the role of the trauma surgeon has been limited recently, since many of the new management tools of the injured consist of selective nonoperative techniques. In 2005, the concept of "Acute Care Surgery" as a new specialty was defined, and that is how the landscape in North America changed. ${ }^{6}$ While this was happening, other great things occurred: in 2006, Dr Juan Carlos Puyana of the University of Pittsburgh, in conjunction with the University del Valle, managed to obtain a Fogarty trauma research grant for USD 750,000. ${ }^{7}$ In addition, Dr Alberto García and I had the fortune of visiting Puyana's team in Pittsburgh and learned about trauma research. In total, nine people from Cali had the opportunity to receive training in research. Since then, we set goals that have been fully met: the submission and acceptance of oral and posters presentations consecutively to the annual congresses of the American Association for the Surgery of Trauma (AAST) which is considered one of the most important scientific meetings in trauma of the USA and the world. The goal was to showcase our experience and contributions to the management of trauma in Colombia (Fig. 2).

In 2005, the American Trauma Committee of the American College of Surgeons (AAST and ACS) formalized the initiative to create "Acute Care Surgery" and, later in 2008, between these two institutions, the fellowship in Acute Care Surgery was founded. ${ }^{6}$ Similarly in Cali, in 2007, we created in conjunction with University del Valle a formal curriculum for the first "Trauma and Emergency Surgery Fellowship Program" in Colombia and had full national verification in 2010 with the enrollment of our first Fellow. This program has had full support since its inception from the following institutions: University Hospital del Valle "Evaristo García" and the Foundation Valle del Lili. The pillars of the Trauma Surgery Fellowship Program are the same as those of the ACS program of "Acute Care Surgery" in the USA. The program is based on trauma, emergency surgery, the management of the patient in the intensive care unit, elective general surgery, and, now, the new concept of "rescue surgery," which encompasses acute, complicated nontraumatic pathology from other specialties. Acute care surgeons have a unique ability to handle and save these patients (Fig. 3). ${ }^{8}$ The first surgeons

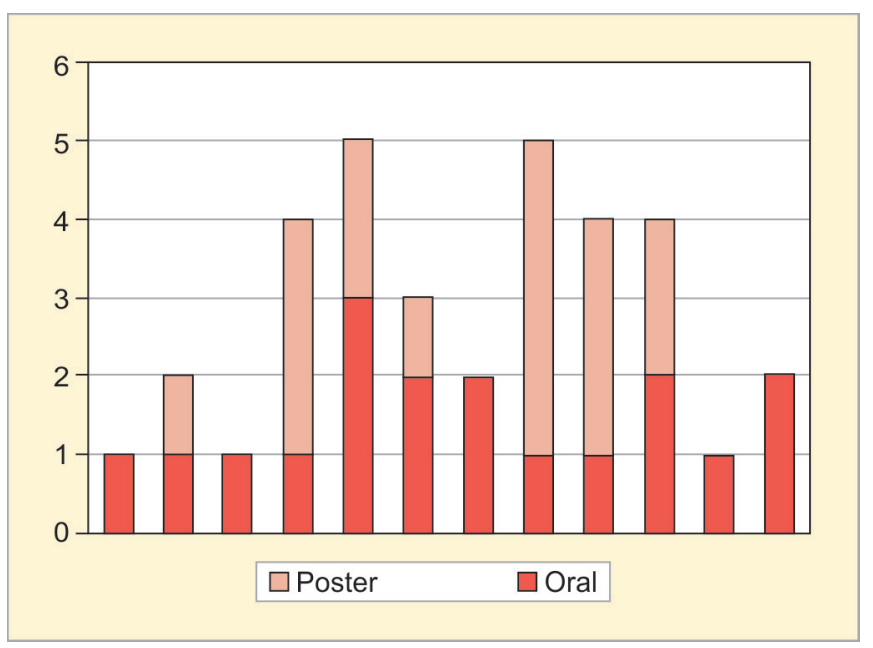

Fig. 2: Trend of the number of abstracts presented at the Annual Meeting of the American Association for the Surgery of Trauma (AAST) (poster and oral presentations) 


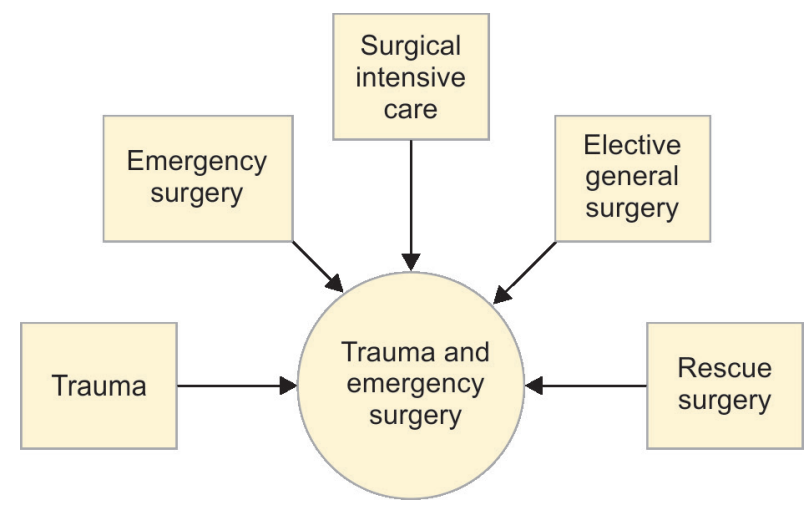

Fig. 3: Pillars of the trauma and emergency surgery

to receive the title of surgeons of Trauma and Emergency Surgeons were Laureano Quintero, Ricardo Ferrada, and I; as professors of the Department of Surgery from the del Valle University, followed by Dr's Luis Fernando Pino (Colombia) and Juan Carlos Salamea (Ecuador). What is special and different about a surgeon of trauma and emergency is that he possesses all the skills to manage trauma patients in the operating room, in the ICU, and also able to perform and manage acute care surgery patients. $\mathrm{He}$ is also capable of teaching these skills to others within Colombia and/or their countries of origin. In addition to this, he or she has the skills to carry out research to generate new knowledge and the leadership backbone to build a trauma and emergency service from scratch.

The Panamerican Society of Trauma has an excellent trauma registry, which has been renewed and is available to anyone interested in research. Dr Rao Ivatury and Dr Michel B. Aboutanos had an important role in the creation of this tool. The PTS provides it to all those who are interested. To achieve a deep and appropriate understanding of the research, collaborative efforts are needed. An example of such a collaboration is since 2011, Dr Michael W. Parra from Fort Lauderdale, Florida, has closely worked with our team in Cali in the conceptualization and writing of many research projects. These types of alliances are important, since they have allowed us to understand that, for us to be able to transmit our ideas, we need first someone who can understand them within our Latin context, but simultaneously, have the ability to transmit them to the World. Similarly, since 2013, we have collaborated with the World Society of Emergency Surgery in their multicenter studies and management guidelines. Thanks to these alliances, we have had the opportunity to participate and publish in these important global societies. All this has given us results in research and publications. Since we started these strategic alliances, the number of publications has progressively increased and the qualities of the manuscripts have also increased exponentially, allowing us to publish in recognized scientific journals around the World. We see this as an opportunity to transmit knowledge to surgeons located anywhere around the globe based on a volume of trauma cases seldom seen anywhere else (Fig. 4). The del Valle University Hospital and the Foundation Valle del Lili are the two university hospitals who have provided support to the program of Trauma and Emergency Surgery. In addition, they have provided economic support to the fellows, who can receive income when taking on-call shifts in the ICU and general surgery ER coverage, providing economic stability while they complete their training. The fellows of Trauma and Emergency Surgery, Juan Carlos Salamea and Luis Fernando Pino, were the first to have the opportunity to go to clinical rotations to Pittsburgh, with the support of Juan Carlos Puyana and Andrew B. Peiztman and the Virginia Commonwealth University in Richmond with Rao Ivatury and Michel B. Aboutanos. This support has been possible in the United States thanks to the Panamerican Trauma Society. Years ago, having the opportunity to do international rotations during our surgical residency here in Colombia was unthinkable. In Richmond, VA, the director of the EMS system Robert Lawrence has offered an opportunity to our fellows to participate and learn about the prehospital/paramedic management of a citywide medical emergency system. Similarly, they have had the opportunity to receive training in advance laparoscopic, trauma, and robotic surgery with Dr Michael W. Parra. Also, Dr Gregory Peck's group at Rutgers University in New Jersey has supported our trauma training program. Dr Thomas Scalea, at the Shock Trauma Center, Baltimore, MD, has graciously opened the doors to our fellows. Generally, a weekly rotation program costs approximately USD $\$ 5,000$, but thanks to our integration with this PTS, the rotation is completely free. We have successfully trained a total of 28 fellows: Colombia (20), Ecuador (5), Mexico (1), Chile (1), and Costa Rica (1). There is a need to create similar new training programs throughout Latin America. Our group is totally willing to collaborate and promote this process; soon we will have a new school in Medellín, and we are in the process of creating and promoting trauma schools in Brazil. Once again, I want to reference the extensive work that

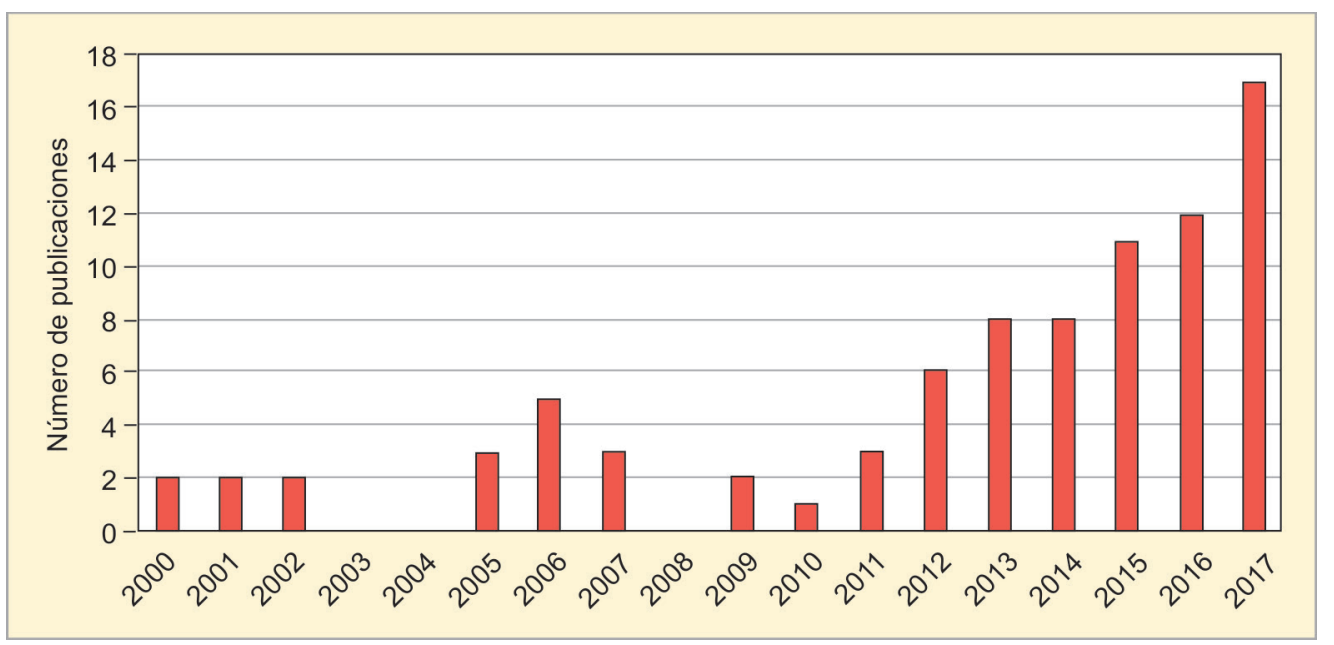

Fig. 4: Trend of the number of publications in indexed journals per year 


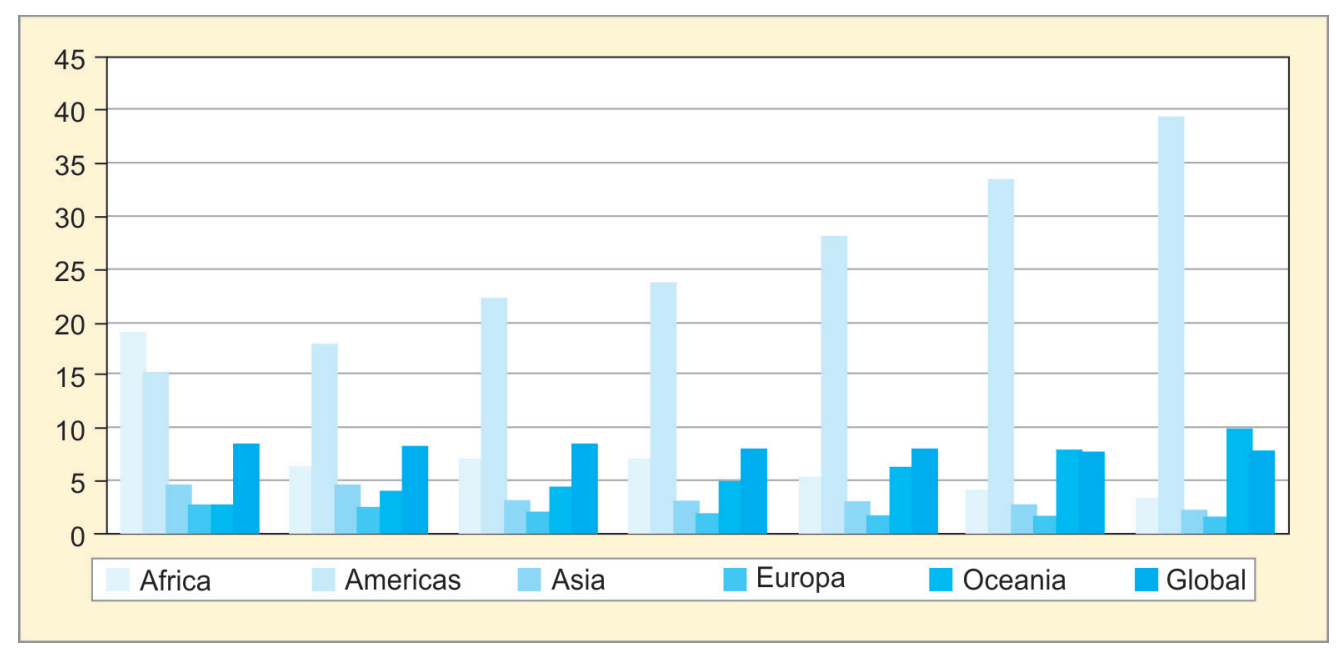

Fig. 5: Projection of the homicide rate in the Americas, from 2000 to 2030: homicide rate per 100.000 habitants

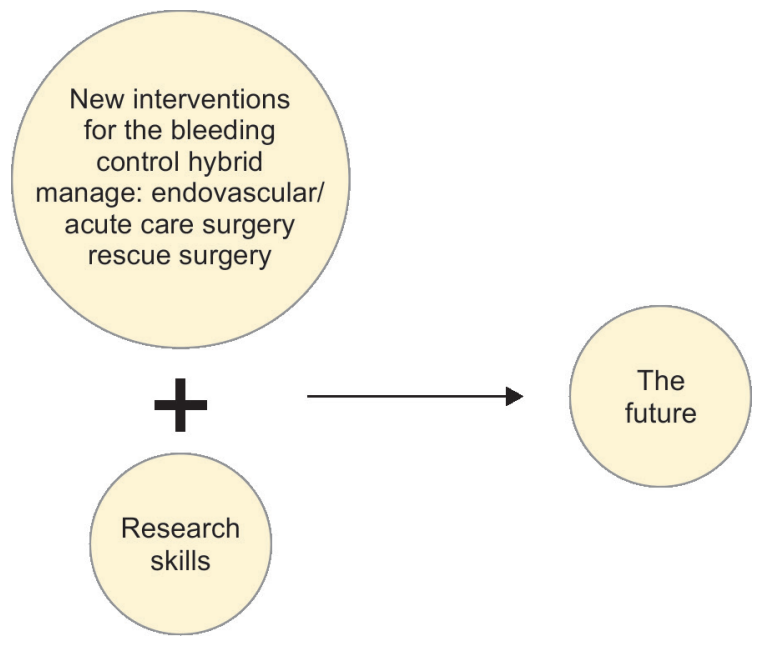

Fig. 6: Skills of the future trauma and emergency surgeon

one of our fellowship graduates Dr Juan Carlos Salamea has been able to create and consolidate a trauma team, trauma league, and a regional trauma system in his home town of Cuenca, Ecuador.

\section{The Future}

In the Americas, according to the World Economic Forum, the trend of the homicide rate for every 100,000 inhabitants will increase significantly to an estimated rate of 40 per every 100,000 inhabitants. We have to prepare to face this challenge (Fig. 5). Our pragmatic strategy is to save lives, we have to innovate and anticipate the future with new interventions that can stop quickly and efficiently traumatic bleeding: hybrid rooms, intravascular management, rescue surgery, damage control, in combination with research skills, to be able to quickly transmit knowledge, and achieve more and more progress (Fig. 6).

A clear example of the application of this innovative strategy "Stop the Bleed" was that of a patient that was managed at the Valle del Lili Foundation, with complex hepatic trauma, who benefited from hybrid interventions. Attended by a team trained in trauma, the patient underwent angiography and embolization of the right hepatic artery. Since he was still in shock, an endovascular Resuscitative Balloon Occlusion of the Aorta (REBOA) was placed.
Despite this, he continued with hemodynamic instability, so another REBOA in the proximal Vena Cava was placed and he underwent a laparotomy to manage liver bleeding. ${ }^{9}$ With an intra-operative Pringle maneuver to complete the liver isolation initiated by the REBOA and REBOC (resuscitative endovascular occlusion of the vena cava), we were able to successfully control the surgical bleeding from the liver. Toward this innovation strategy is to what we want to point to and I had the opportunity to share this case with surgeons of trauma in Japan. There, a hybrid room in the emergency department is standard of care, and studies have shown a significant decrease in the overall mortality of patients who have benefitted from this technology as compared to the conventional trauma bay-ER rooms.

The traditional general surgeon is slowly disappearing, but the Acute Care Trauma surgeon has resurrected with broad new strategies: trauma surgery, medical-surgical emergencies, rescue surgery, elective surgery, with deep knowledge of surgical intensive care, with the ability to educate future generations, carry out research, produce scientific knowledge, and lead and build future trauma and emergency centers. Now, we have a novel idea to improve even more the training of future trauma and emergencies surgeons. Juan Pablo Herrera studied 3 years in research with the group of Dr Adil Haider in Boston, MA. Through a strategic alliance with the Panamerican Society of Trauma, currently, he has had the opportunity to present trauma research at the AAST Annual Congress, without even being a surgeon yet. The idea is to obtain a graduate degree in public health, then a general surgery residency program, and, finally, complete your training by completing a Fellowship in Acute Care Trauma Surgery. Currently, we are preparing trauma surgeons as described. The trauma and emergency surgeons must master all surgical techniques, have the ability to investigate, and convert what has been learned in education and innovation. They will be the new "MacGyver's" of the world, able to face anything and everything, with the clear objective of saving lives in their hospitals and in the world (Fig. 7).

This conference is dedicated to Dr Ricardo Ferrada, who initiated the process of creating surgeons of trauma and emergency in Latin America, to Dr Rao Ivatury, for having dedicated most of his life to encourage this true union of the Americas through the Panamerican Trauma Society, to Dr Aurelio Rodríguez, creator of the Panamerican Trauma Society that has achieved the integration that we have today and, finally, to the members and the soul of what today is the great organization: Sociedad Panamericana de Trauma. 


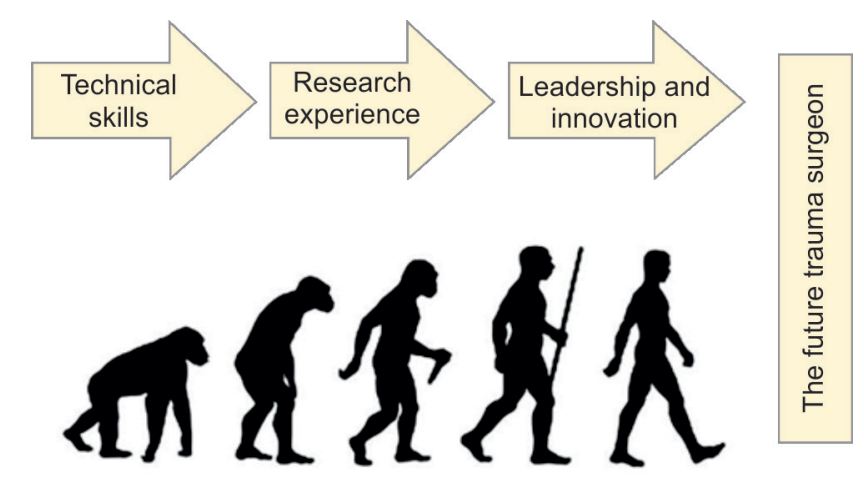

Fig. 7: Model of the evolution to become a future trauma and emergency surgeon

\section{References}

1. Ivatury RR, Aboutanos M. Panamerican trauma society: the first three decades. J Trauma Acute Care Surg 2017;82:966-973. DOI: 10.1097/ TA.0000000000001393.
2. Borráez O. Open abdomen: the most challenging wound. Rev Colomb Cir 2008;23:204-209.

3. Chatterjee K. The Swan-Ganz catheters: past, present, and future. Circulation 2009;119:147-152.DOI: 10.1161/CIRCULATIONAHA.108.811141.

4. Rotondo MF, Schwab CW, et al. Damage control: an approach for improved survival in exsanguinating penetrating abdominal injury. J Trauma 1993;35:375-383. DOI: 10.1097/TA.0b013e3181e12b3a.

5. Barker DE, Kaufman HJ, et al. Vacuum pack technique of temporary abdominal closure: a 7 year experience with 112 patients. J Trauma Acute Care Surg 2000;48:201-207.

6. Davis KA, Jurkovich GJ. Fellowship training in acute care surgery: from inception to current state. Trauma Surg Acute Care Open 2016;1:1-5. DOI: 10.1136/tsaco-2016-000004.

7. Sánchez-Ortiz A. Mortality and costs from acute care surgical emergencies. University of Pittsburgh; 2010 Date of consultation: September 2017. Available at: http://d-scholarship.pitt.edu/25071/1/ SanchezAI_ETD_Pitt2015.pdf.

8. Kutcher ME, Sperry JL, et al. Surgical rescue: the next pillar of acute care surgery. J Trauma Acute Care Surg 2017;82:280-286. DOI: 10.1097/ TA.0000000000001312.

9. Ordóñez CA, Herrera-Escobar JP, et al. A severe traumatic juxtahepatic blunt venous injury. J Trauma Acute Care Surg 2016;80:674-676. DOI: 10.1097/TA.0000000000000979. 